\title{
Negative Refractive Index Shaped Lens for Mobile Base Station
}

\author{
Salbiah Ab Hamid, Nurul Huda Abd Rahman, Yoshihide Yamada, and Idnin Pasya Ibrahim
}

\begin{abstract}
Recently, there are rapid developments in antenna researches to fulfil the advanced mobile technology requirement including 5G technology. Narrow beam width, higher frequency, and higher gain are among the key characteristics in $5 \mathrm{G}$ antenna technology to serve a massive number of users, consistent interconnectivity, and higher capacity. Previously, the multi-beam operation was performed by using array antennas, however, in the 5G system, more losses due to complex feeding network is expected if the conventional array antenna is adopted. Therefore, the lens antenna is identified as one of the potential candidates to replace the current antenna structure used in the mobile base station. In this study, the characteristics of the negative refractive index shaped lens using the energy conservation law in mobile base station applications are investigated. One of the significant findings from this study is that a smaller radius and slender lens configuration can be achieved using a negative refractive index. A lens antenna with the gain of $27.55 \mathrm{~dB}$ and narrow beam width of $7.81^{\circ}$ is produced. This configuration is suitable for mobile base station application due to its less bulky characteristics which makes it easy to be mounted on the base station.
\end{abstract}

Index Terms-5G, mobile base station, lens antenna, negative refractive index

\section{INTRODUCTION}

$\mathrm{T}$ OWARDS 5G implementation, there are a few key elements to be considered including system requirement and antenna characteristics. 5G technology demands for millimeter wave frequency range and massive Multiple Input Multiple Output (MIMO) for signal processing. Furthermore, digital beam forming (DBF) multi beam and beam steering are expected for the antenna system. Currently, for mobile base station application linear arrays are used as illustrated in Fig. 1(a). Linear array is divided into a number of sub arrays to reduce the feeding cable. For higher frequency application, due to the reduction of antenna size and beam coverage, this structure is no longer the best candidate because more arrays and feeding cable are needed which will contribute to higher feeder losses as well as result in more complex structure.

This manuscript is submitted on 3th Spetember 2020 and accepted on 5th February 2021.This work was supported by Antenna Research Centre (ARC), Faculty of Electrical Engineering, Universiti Teknologi MARA (UiTM) Shah Alam, Malaysia while Salbiah Ab Hamid and Nurul Huda Abd Rahman are with ARC (salbiaha22@gmail.com, nurulhuda0340@uitm.edu.my). Idnin Pasya Ibrahim is with Microwave Research Institute (MRI), UiTM and Yoshihide Yamada is with Malaysia Japan International Institute of Technology (MJIIT), Universiti Teknologi Malaysia, Malaysia (ndayamada@yahoo.co.jp)

1985-5389/C 2021 The Authors. Published by UiTM Press. This is an open 94 access article under the CC BY-NC-ND license (http://creativecommons.org/ licenses/by-nc-nd/4.0/).
A larger space is needed to allocate the complex feeding network, and finally this will increase the radome size. In order to reduce the complexity, the usage of a single feed to replace the array feed is proposed as shown in Fig. 1(b). Lens is added to the base station radome in order to enhance the radiated signal power. However, the lens thickness becomes the main concern. In order to produce optimum lens performance, positive refractive index lens, $\mathrm{n}>0$ will give higher lens thickness which is not suitable for installation. Therefore, negative refractive index lens, $\mathrm{n}<0$ is proposed and studied in this paper.

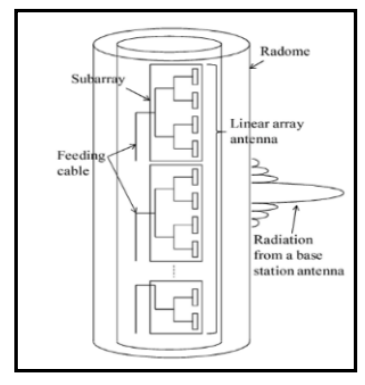

(a) Present base station configuration

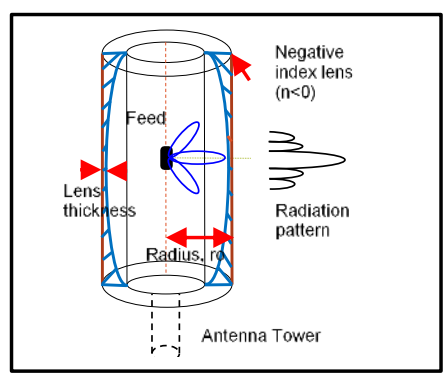

(b) Proposed base station (single feed)
Fig.1. Mobile Base Station

The proposed lens antenna system consist of feed radiators and the dielectric lens. The feed can be of many types of antennas such as microstrip patch, horn or array antenna. Lens is used to direct or collimate the radiated waves from the feed. Specifically, lens will convert the spherical wave from the feed into plane wave at the aperture [1]. In lens antenna application, lens is expected to improve the antenna performance in terms of gain as well as the beam width. Although dielectric lens antenna have been widely used, the negative refractive indexed or also known as metamaterial lens concept is quite new. As for metamaterial antennas, some related works have been performed. A high gain of antipodal Vivaldi antenna with metamaterial covers with operating frequency band of $0.95 \mathrm{GHz}$ $-11 \mathrm{GHz}$ was designed by [2].The antenna gain is improved from $6.86 \mathrm{~dB}$ to $17.67 \mathrm{~dB}$. This high gain, narrow beam width and broad bandwidth antenna is suitable for ground penetrating radar, microwave imaging and other broadband wireless application. Previous studies reveals that metamaterial lens antenna is also improves antenna directivity [3]. A metamaterial 
unit cell with $12 \mathrm{GHz}$ operating frequency is designed and converted into a periodic layer. The performance of one layer and two layers of periodic cell were studied. A microstrip antenna directivity was significantly improved by inverse refraction of the proposed metamaterial with $2.74 \mathrm{~dB}$ and 4.08 $\mathrm{dB}$ respectively. The same trend was observed in Ultra-Wide Band (UWB) antenna where a single array layer with $3 \times 2$ inverted L-shaped metamaterial unit cells was added thus enhancing the gain due to the negative characteristics of the metamaterial structure which behaves as super lens when it was placed in front of the antenna as a cover. [4]

In [5], an ellipsoid lens fed by phased array antenna is proposed in order to solve the issues on conventional Luneburg lens antenna. The proposed ellipsoid lens is reduced by half compared to conventional Luneburg lens. The wide scanning property is also obtained by optimizing the amplitude and phase distribution of phased array antenna (PAA). The proposed lens gives the maximum gain of $21.74 \mathrm{~dB}$.

In this paper, a negative refractive index shaped lens with small thickness and smaller radius size is proposed. This configuration offers a high gain and produces narrow beam width with operating frequency of $28 \mathrm{GHz}$. This paper is organized into four sections. Section I is for the introduction part. Section II described the design method and in section III, shaped lens analysis is demonstrated. In section IV, some concluding remarks are provided.

\section{DESIGN METHOD}

Fig.2 illustrates the proposed lens antenna parameter configuration. At surface $1, S_{1}$ and surface $2, S_{2}$ of the lens, Snell's law is applied using equation (1) and (2) respectively. The parameter descriptions are summarized in TABLE I. The lens used in this study is negative refractive index, $n<0$.

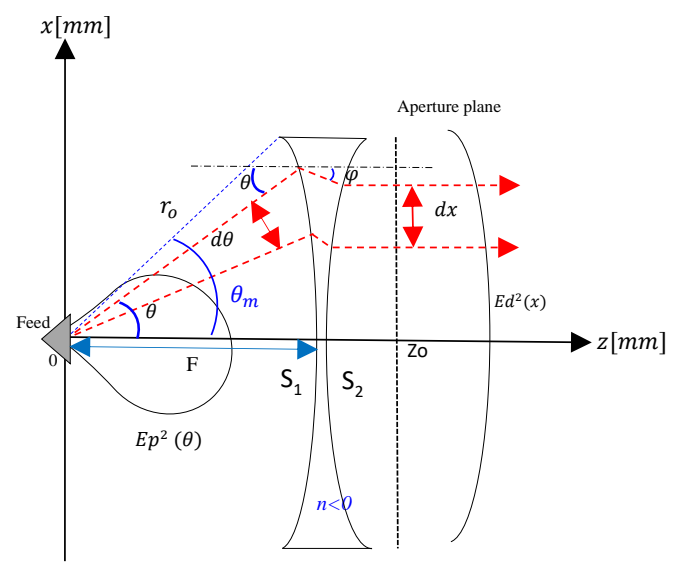

Fig. 2. Lens Configuration and Parameters
TABLE I

DESIGN SPECIFICATIONS OF PROPOSED ANTENNA PARAMETER

\begin{tabular}{|l|c|}
\hline \multicolumn{1}{|c|}{ Parameters } & Descriptions \\
\hline$n$ & Refractive index $(\mathrm{n}<0)$ \\
\hline$E p^{2}(\theta)$ & Feed pattern \\
\hline$E d^{2}(x)$ & Aperture Distribution \\
\hline$\theta m$ & Angle from feed to the lens edge \\
\hline$\theta s$ & Beam shift angle \\
\hline
\end{tabular}

$\frac{d r}{d \theta}=\frac{r n \sin (\theta-\varphi)}{n \cos (\theta-\varphi)-1}$

At the lens surface 2, the expression for the slope $\frac{\mathrm{dz}}{\mathrm{dx}}$ can be derived from the condition that all exit rays after refraction are parallel to the $\mathrm{z}$ - axis is shown in Eq. (2). The $\frac{\mathrm{dz}}{\mathrm{dx}}$ expression can be separated into $\frac{d z}{d \theta}$ and $\frac{d x}{d \theta}$ shown in Eq. (2) for variable change from $\mathrm{dx}$ to $\mathrm{d} \theta$.

$\frac{d z}{d x}=\frac{n \sin (\varphi)}{1-n \cos (\varphi)} \quad, \frac{d z}{d \theta}=\frac{n \sin (\varphi)}{1-n \cos (\varphi)} \frac{d x}{d \theta}$

By using this equation, the variable $\varphi$ in Eq. (1) and (2) can be expressed by the variable $\theta$. Then, through simplification, the variable of Eq. (1) and (2) becomes only $\theta$. The electric power conservation at the ray is composed of $d x$ and $d \theta$

$$
\frac{d x}{d \theta}=\frac{E p^{2}(\theta)}{\int_{0}^{\theta m} E p^{2}(\theta) d \theta} \frac{\int_{0}^{X m} E d^{2}(x) d x}{E d^{2}(x)}
$$

For the feed pattern, $\operatorname{Ep}^{2}(\theta)$,equation (4) is implemented. The concept of $\operatorname{Ep}^{2}(\theta)$ is shown in Fig.3 (a). Meanwhile, equation (5) is used for aperture illumination distribution, $E d^{2}(x)$ shown in Fig.3 (b).

$E p^{2}(\theta)=\cos ^{m}(\theta-\theta s)$
$E d^{2}(x)=\left[\left(1-\left(1-\frac{1}{c}\right)\left(\frac{x}{x_{m}}\right)^{2}\right)\right]^{\mathrm{p}}$

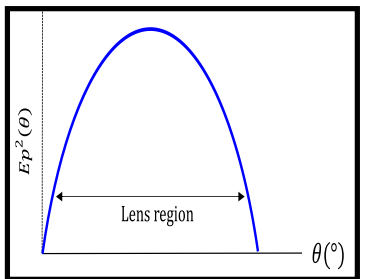

a) Feed pattern, $E p^{2}(\theta)$

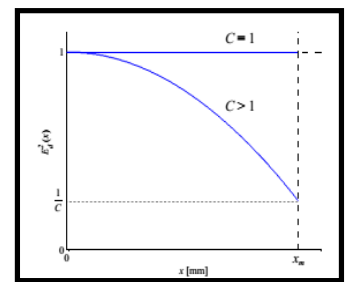

b) Aperture distribution, $E d^{2}(x)$
Fig. 3. MATLAB Parameters

A flow chart in Fig. 4 represents the processes involved in negative refractive index lens design using MATLAB software. Program codes were developed based on the equations and formula that were previously described. The initial parameters $\left(\mathrm{n}, \theta_{\mathrm{m}}, \mathrm{r}_{\mathrm{o}}, \mathrm{d}_{\mathrm{o}}\right.$ ) determined at the lens edge as shown in Figure 5. The equations for feed radiation pattern, $\operatorname{Ep}^{2}(\theta)$ and aperture 
distributions, $E d^{2}(x)$ are given. Next, differential equations (1), (2) and (3) are solved by the Matlab routine of "ode45".

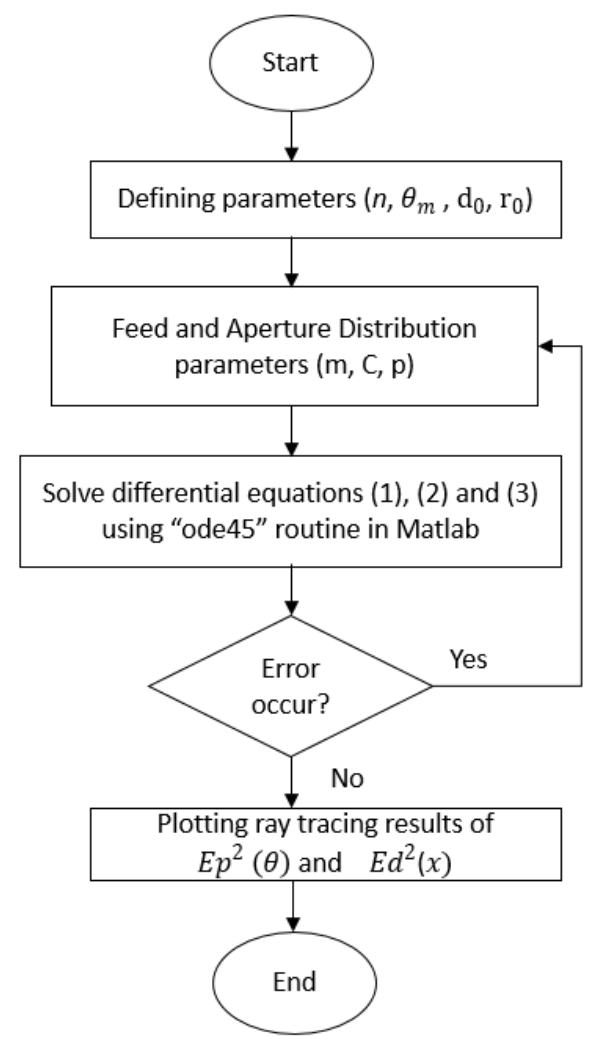

Fig.4. Design Flow Chart

Explanation for the initial input values is shown in Figure 5. $\theta_{0}$ is an important parameter that determine the lens thickness where $\mathrm{d}_{0}$ is the initial thickness.

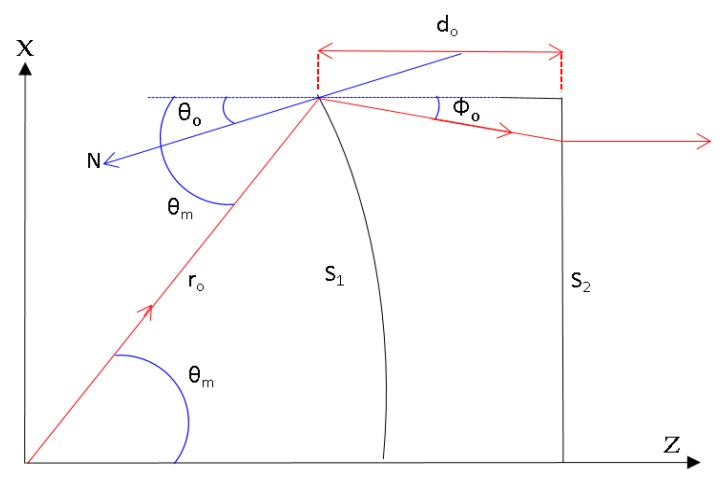

Fig. 5. Initial parameters at the lens edge

\section{SHAPED LENS DESIGN}

The shaped lens design process is performed using MATLAB software considering the equations stated in section II. The main concern in the design process is to obtain the slender lens and the smallest radius indicated by the distance between the feed and lens. Some examples of the shaped lens are tabulated in Table II and Table III. Table II represents the shaped lens using positive refractive index while Table III represents the shaped lens using a negative refractive index. Three different angles with two different refractive indexes are selected in this paper to show several possible shapes designs.

TABLE II

EXAMPLE OF POSITIVE REFRACTIVE INDEX SHAPED LENS

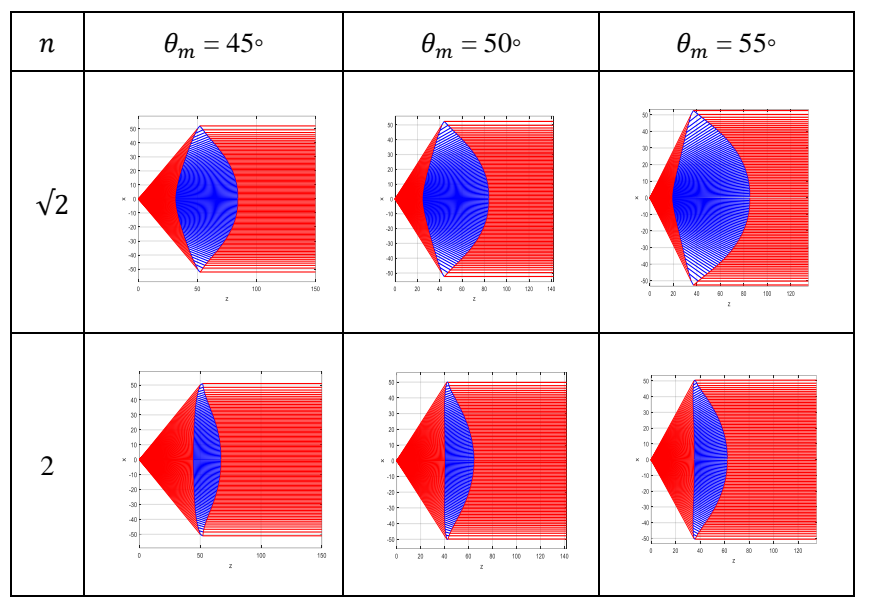

For the positive refractive index design in Table II, the lens thickness becomes smaller as the refractive index increases. On the other hand, the lens radius is getting smaller when the maximum angle from the feed to the edge of the lens, $\theta_{m}$ increases. The smallest radius is around $60 \mathrm{~mm}$.

TABLE III

EXAMPLE OF NEGATIVE REFRACTIVE INDEX SHAPED LENS

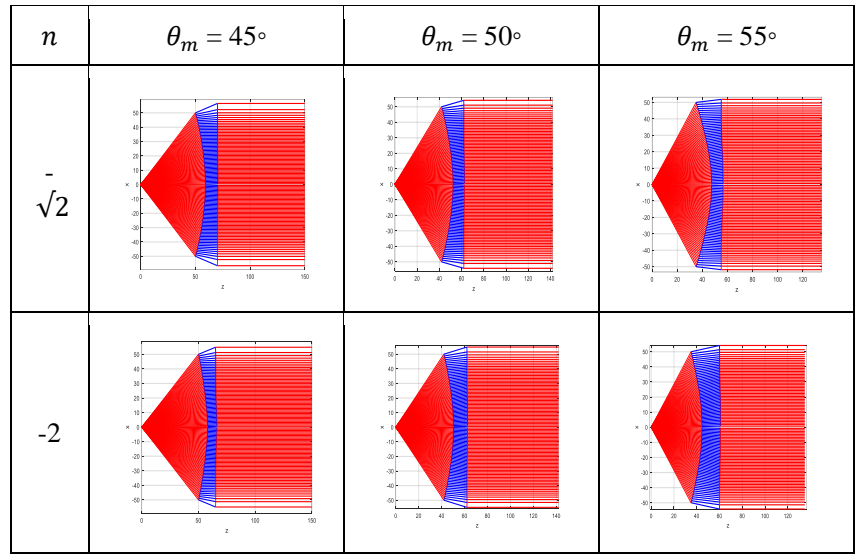

In the case of negative refractive index design in Table III, the smallest radius obtained is around $50 \mathrm{~mm}$. Smaller lens thickness can be achieved as the refractive index becomes more negative. The negative refractive index gives a slender lens as compared to the positive refractive index. Both small lens thickness and small radius characteristics are important for practical installation purposes. In order to evaluate the performance of the designed lens, the lens structure is translated into an EM simulator, HFSS. In MATLAB, a point source was used to represent the feed, but in HFSS, a horn antenna is used to represent the feed radiator as shown in Fig.6 (a). The performance of the horn feed are presented in Fig.6 (b) and 
(c).The gain is $10.689 \mathrm{~dB}$ with $56.03^{\circ}$ beamwidth, $\theta_{B}$ for both E-Plane and H-Plane.

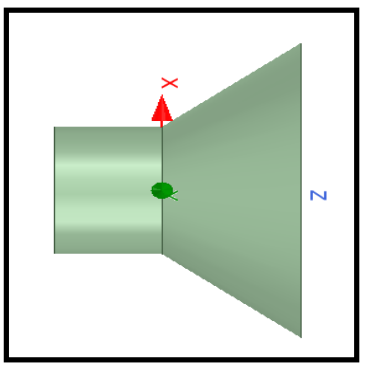

(a) Horn Structure

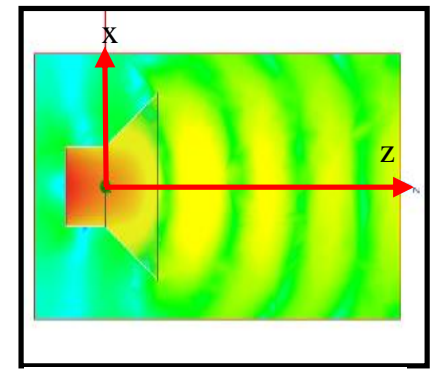

(b) Electric Field

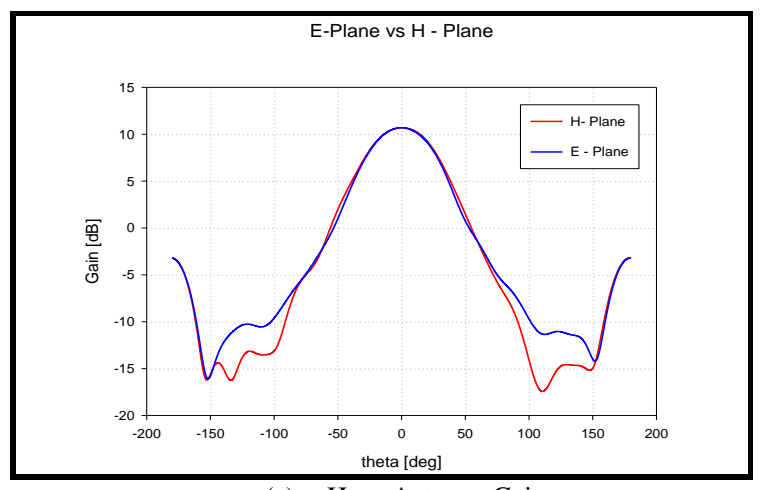

(c) Horn Antenna Gain

Fig.6. Horn Antenna Performance

Due to the smallest lens thickness shown by $n=-\sqrt{2}$ and $\theta_{m}$ $=55^{\circ}$ in Table III, lens structure in Fig. 7(a) is selected to be designed in HFSS with following parameters in Table IV. Fig. 7(b) shows the lens structure in HFSS.

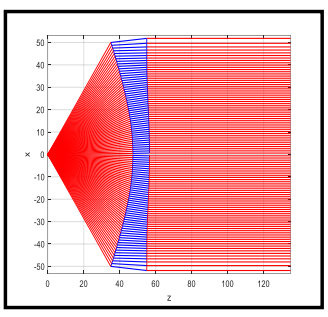

a) Selected Shape

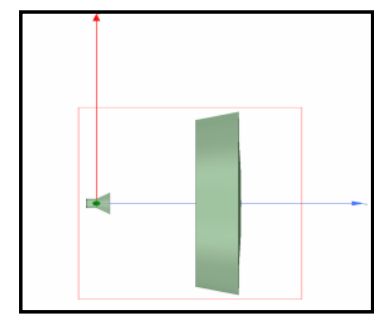

b) Lens antenna in HFSS
Fig.7. Negative Refractive Index Shaped Lens

TABLE IV

LENS SIMULATION PARAMETERS

\begin{tabular}{|c|c|}
\hline Parameters & Descriptions \\
\hline$\mu_{\mathrm{r}}$ & -2 \\
\hline$\varepsilon_{\mathrm{r}}$ & -1 \\
\hline Frequency $(\mathrm{GHz})$ & 28 \\
\hline Lens Diameter $(\mathrm{mm})$ & 100 \\
\hline
\end{tabular}

Simulation results for the negative refractive index shaped lens are described in Fig. 8 and Fig.9 respectively. It is observed that the lens produced the gain of $27.55 \mathrm{~dB}$. The beamwidth becomes narrow from $56.03^{\circ}$ to $7.81^{\circ}$. The performance of the designed lens is summarized in Table V. This lens performs with 66\% efficiency. From Fig.9, it can be observed that lens antenna converts the spherical wave from the feed radiator into a plane wave at the aperture.

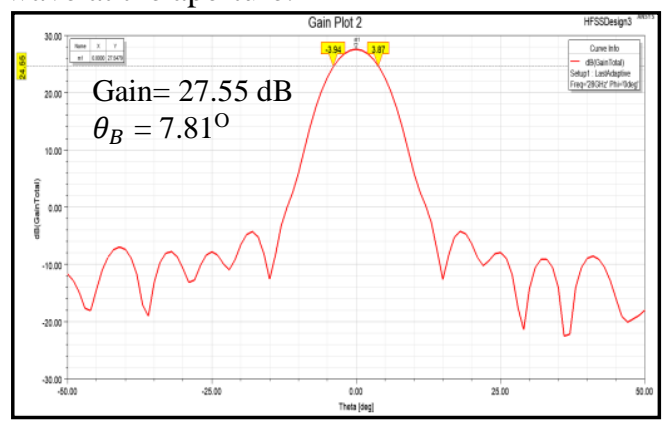

Fig. 8. Antenna gain after lens added

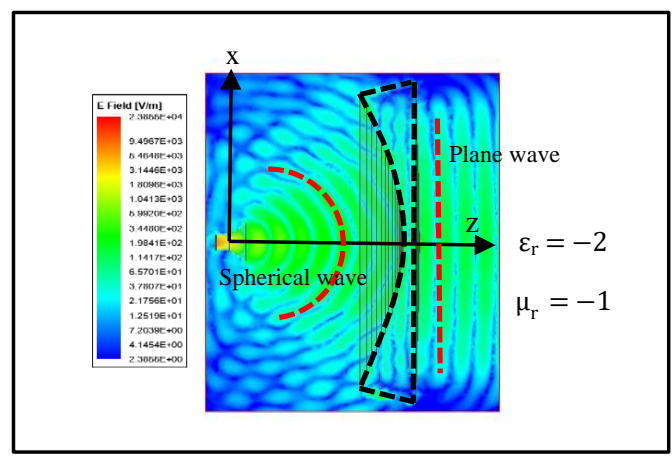

Fig. 9. Electric field distribution

TABLE V

LENS PERFORMANCE

\begin{tabular}{|l|c|c|}
\hline & Theoretical & Simulation \\
\hline Gain $(\mathrm{dB})$ & 29.35 & 27.55 \\
\hline$\theta_{B}\left({ }^{\circ}\right)$ & 8.01 & 7.81 \\
\hline$\Delta G(\mathrm{~dB})$ & 0 & -1.80 \\
\hline $\boldsymbol{\eta}(\%)$ & 100 & 66 \\
\hline
\end{tabular}

The electric distribution in magnitude and phase are shown in Fig. 10(a) and (b) respectively, performed through near-field calculation. For on-focus condition, both intensity and phase distributions show uniform and symmetrical characteristics.

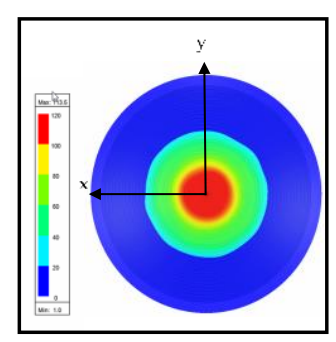

a) Magnitude distribution

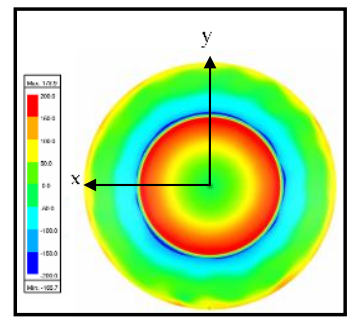

b) Phase distribution Fig. 10. Near field results

\section{CONCLUSION}

The performance of the negative refractive index shaped lens for future 5G mobile base station application was evaluated in 
this paper. The negative refractive index lens offers smaller lens thickness as compared to positive refractive index lens which is suitable for installation. Overall simulation results of the proposed lens structure show good agreement with theoretical results. The designed shaped lens increases the antenna gain and narrowing the beamwidth. Nevertheless, high gain and narrow beamwidth are two key factors for 5G technology.

\section{ACKNOWLEDGEMENT}

The authors would like to give appreciation towards Antenna Research Centre (ARC), Faculty of Electrical Engineering, and Universiti Teknologi MARA (UiTM) Shah Alam for providing facilities to conduct the present research and to Malaysia Ministry of Higher Education for supporting this project through Grant Scheme FRGS/1/2018/TK04/UITM/02/25.

\section{REFERENCES}

[1] Y.T. Low/S.W. Lee, Antenna Handbook, Van Nostrand Reinhold; Volume II, pp. 16-5-16-50.

[2] High-gain antipodal Vivaldi antenna with metamaterial covers, IET Microwaves, Antennas \& Propagation,2019

[3] Urul B. Gain enhancement of microstrip antenna with a novel DNG material. Microw Opt Technol Lett. 2020;1-6.

[4] Wael A. E. Al;Gain improvement of tunable band-notched UWB antenna using metamaterial lens for high speed wireless communications,Microsystem Technologies, 2019, volume 25, Issue 11, pp 4111-4117.

[5] Liu K, Yang S, Chen C, Qu S-W, Chen Y. A wide-scanning ellipsoid lens antenna fed by phased array antenna. Int J RF Microw Comput Aided Eng. 2020;e22127.

[6] Tajima, Y.; Yamada, Y. Design of Shaped Dielectric Lens Antenna

[7] Michishita, N.; Yamada, Y. Metamaterial Radome Composed of Negative Refractive Index Lens for Mobile Base Station Antennas, Proceedings of the International Conference on Advanced Technologies for Communications, Hanoi, Vietnam, 15-17 Oct. 2014.

[8] Ziolkowski, R.W. Design, fabrication, and testing of double negative metamaterials. IEEE Trans. Antennas Propag.2003, vol. 51, pp. 15161529 .

[9] Tajima, Y.; Yamada, Y. Simulations of a Shaped Dielectric Lens Antenna by FEKO. ACES journal.2009, vol. 24, pp. 419-426.

[10] Tajima, Y.; Yamada, Y; Michishita, N. Electrical Performance Estimations for Shaped Dielectric Lens Antenna with Array Feed. ACES journal.2010, vol. 25, pp. 458-467.

[11] Stutzman, W. L.; Thiele, G. A. Aperture Antenna. In Antenna Theory and Design, 3rd ed.; Ashenberg, W.; Publisher: John Wiley \& Sons, Inc, America, 2012; Volume 1, pp. 344-428.

[12] Smith, D.R.; Schurig, D. Electromagnetic wave propagation in media with indefinite permittivity and permeability tensors. Phys. Rev. Lett.2003, vol. 90, 077405.

[13] Milligan, T. A. Lens antenna. In Modern Antenna Design, 2nd ed.; Publisher: John Wiley \& Sons, Inc, America, 2005; Volume 1, pp. 447-451.

[14] Pendry JB. Negative refraction makes a perfect lens. Phys Rev Lett. 2000;85(18):3966.

[15] Engheta, N.; Ziolkowski, R. W. Introduction, history, and selected topics in fundamental theories of metamaterials. In Metamaterials Physics and Engineering Explorations, 1st ed.; Akay, M., Groda, T.G., Newman, M.S.; Publisher: John Wiley \& Sons, Inc, America, 2006; Volume 1, pp. 5-37.

[16] Samuel Silver. Microwave Antenna Theory and Design, McGrawhill, p.394, 1949. 